\title{
The Three-Bite Technique: A Novel Method of Dog Ear Correction
}

\author{
Omar Jaber ${ }^{1}$, Marta Vischio ${ }^{2}$, Angela Faga ${ }^{2,3}$, Giovanni Nicoletti ${ }^{2,3}$ \\ ${ }^{1}$ Plastic Surgery Residency Program, University of Pavia, Pavia; ${ }^{2}$ Plastic and Reconstructive Surgery, Department of Clinical Surgical, \\ Diagnostic and Paediatric Sciences, University of Pavia, Pavia; ${ }^{3}$ Plastic and Reconstructive Surgery Unit, Salvatore Maugeri Research and \\ Care Institute, Pavia, Italy
}

The closure of any circular or asymmetric wound can result in puckering or an excess of tissue known as a 'dog ear'. Understanding the mechanism of dog ear formation is a fundamental requirement necessary to facilitate an appropriate treatment. Many solutions have been reported in the literature, but in all cases, the correction entails the extension of the scar and the sacrifice of the dermal plexus. Here, we propose a novel technique of dog ear correction by using a three-bite suture that sequentially pierces the deep fascial plane and each dog ear's margin, thus allowing for flattening the dog ear by anchoring the overprojecting tissue to the deep plane. The three-bite technique proved to be a fast, easy, and versatile method of immediate dog ear correction without extending the scar, while maintaining a full and complete local skin blood supply.

Keywords Skin / Sutures / Cicatrix / Reconstructive surgical procedures / Surgery, plastic
Correspondence: Giovanni Nicoletti Plastic and Reconstructive Surgery Unit, Salvatore Maugeri Research and Care Institute, University of Pavia, Via Salvatore Maugeri 10, Pavia 27100, Italy

Tel: $+39-0382-592222$

Fax: +39-0382-592220

E-mail: giovanni.nicoletti@unipv.it

We thank Alan Serge McGhee, M.Sc., Glasgow City Council Education Department, for his contribution to the submission of this dissertation.

No potential conflict of interest relevant to this article was reported.

Received: 22 Jun $2014 \bullet$ Revised: 16 Jul 2014 • Accepted: 1 Aug 2014

pISSN: 2234-6163 • elSSN: 2234-6171 • http://dx.doi.org/10.5999/aps.2015.42.2.223 • Arch Plast Surg 2015;42:223-225

\section{INTRODUCTION}

'Dog ears' are a very common problem in plastic surgery practice, and their management is extensively described in the literature.

Here, we propose a new suturing technique for dog ear correction designed specifically for flattening of the over-projecting skin without any need for scar lengthening.

\section{IDEA}

The wound is sutured until a dog ear becomes evident. A threebite absorbable suture is then placed between the deep plane and the dog ear skin margins. The first bite pierces the deep fascial plane along the skin suture axis, a few millimetres distal to the last cutaneous stitch. The second bite pierces the dermis in one dog ear's margin in a subcuticular fashion entering at the level of the deep bite exit and coming out $2 \mathrm{~mm}$ proximal to the dog ear's dome. The third bite is carried out in a subcuticular fashion in the other dog ear's margin and is specular to the second one. The thread is then gently tied, with care taken to evenly distribute all fine skin wrinkles and avoiding any purse-string effect from excessive stitch tension. Such a manoeuvre will allow for anchoring the projecting tissue to the deep fascial plane, thus flattening the dog ear without any need for scar lengthening (Fig. 1A).

The three-bite technique as described above allows the correction of a standing full-cone pattern. A slight technical variation is required for the correction of the asymmetric half lying-cone 
Fig. 1. Two different patterns of dog ear

(A) The three-bite technique applied on the standing full-cone dog ear. (B) The three-bite technique applied on the half lying-cone dog ear.

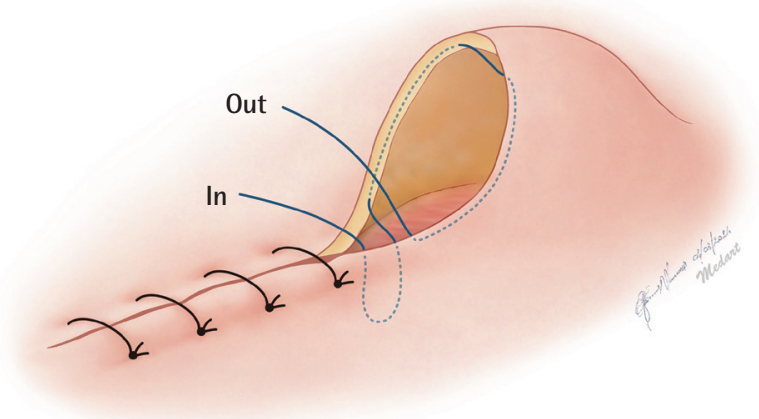

(A)

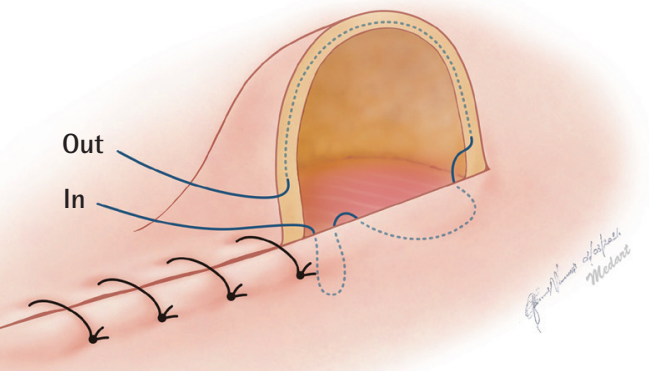

Fig. 2. Case 1, standing full-cone dog ear

Sequenced images of the three-bite technique on the standing full-cone dog ear. (A) Reconstruction with a local skin flap and evidence of the dog ear at the end of the suture line. (B) The suture right after the three-bite technique. (C) The result at the 20-weeks follow-up.
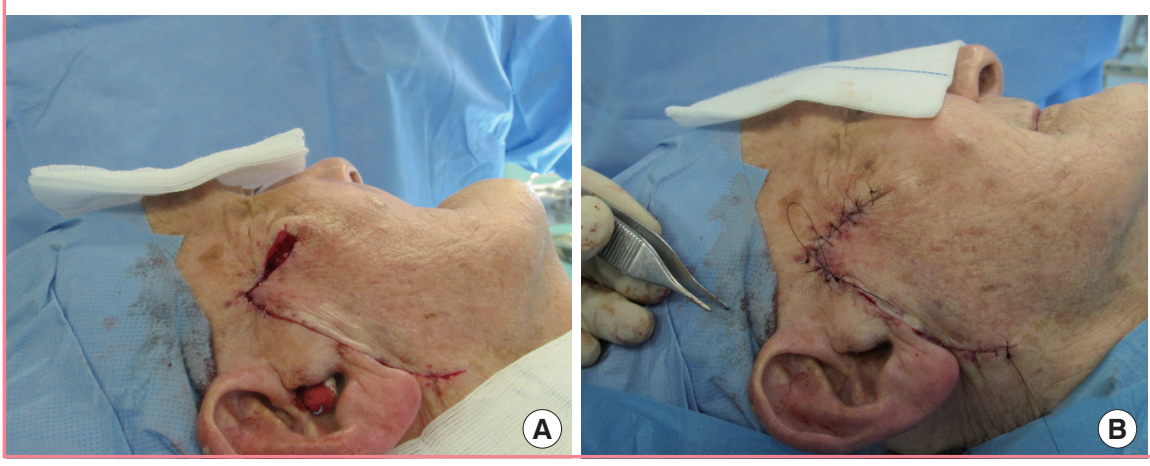

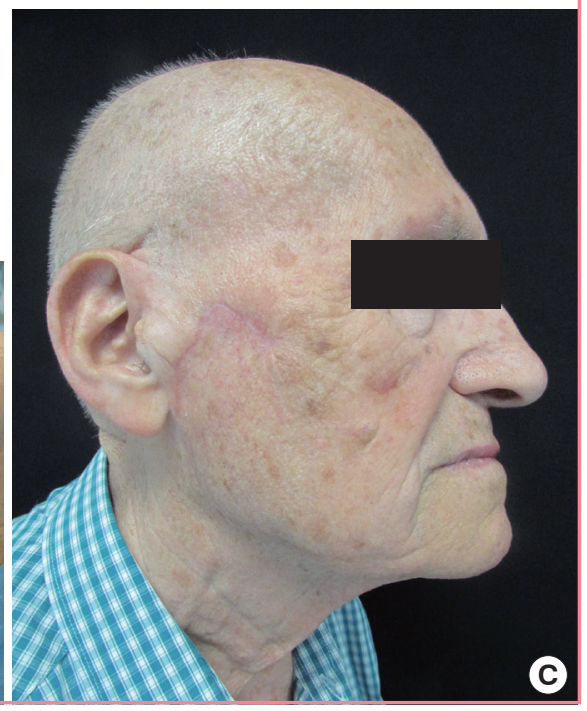

dog ear. The first bite is the same as that described above and pierces the deep fascial plane along the skin suture axis a few millimetres distal to the last cutaneous stitch. The second bite pierces the dermis in the flat skin edge, runs in the dermis along a large bite in a subcuticular fashion, and comes out $2 \mathrm{~mm}$ proximal to the end of the skin incision. The third bite is carried out in a subcuticular fashion along the whole dog ear's margin with the entry and exit sites specular to the opposite skin margin. The fine skin wrinkling due to the approximation of two asymmetric skin margins is evenly distributed while gently tying the stitch (Fig. 1B).

Nine cases of dog ear correction over a sample of 9 patients $(7$ males, 2 females) were carried out at the Plastic and Reconstructive Surgery Unit, University of Pavia, Salvatore Maugeri Research and Care Institute (Pavia, Italy) from January 2014 to March 2014. The patients' mean age was 77 years (minimum age, 64 years; maximum age, 89 years; median age, 72 years). All of the patients underwent excision of the skin tumours of the face (5), scalp (1), and trunk (3), and reconstruction with local skin flaps. In all the cases, the dog ears were easily corrected, and both the patients and the surgeons were very pleased with the eventual outcome (Figs. 2, 3).

\section{DISCUSSION}

Limberg [1] described a dog ear as a conical deformation of tissue. A few years later, Borgess [2] further analysed the shape of a dog ear and described two patterns: the standing full cone and the lying half cone. The former is due to an excess of tissue on both sides of the wound, while the latter is due to an excess of tissue only on one margin of the skin excision.

Tissue dynamics such as elasticity and tension forces, wound geometry, surface contour, and the choice of the most appropriate surgical technique are four major principles of cutaneous surgery, and all of them affect the dog ear formation $[3,4]$. Although we pay great attention to these fundamental techniques, 


\section{Fig. 3. Case 2, half lying-cone dog ear}

Sequenced images of the three-bite technique on the half lying-cone dog ear. (A) Reconstruction with a local skin flap and evidence of the dog ear at the end of the suture line. (B) The suture right after the three-bite technique. (C) The result at the 21-weeks follow-up.
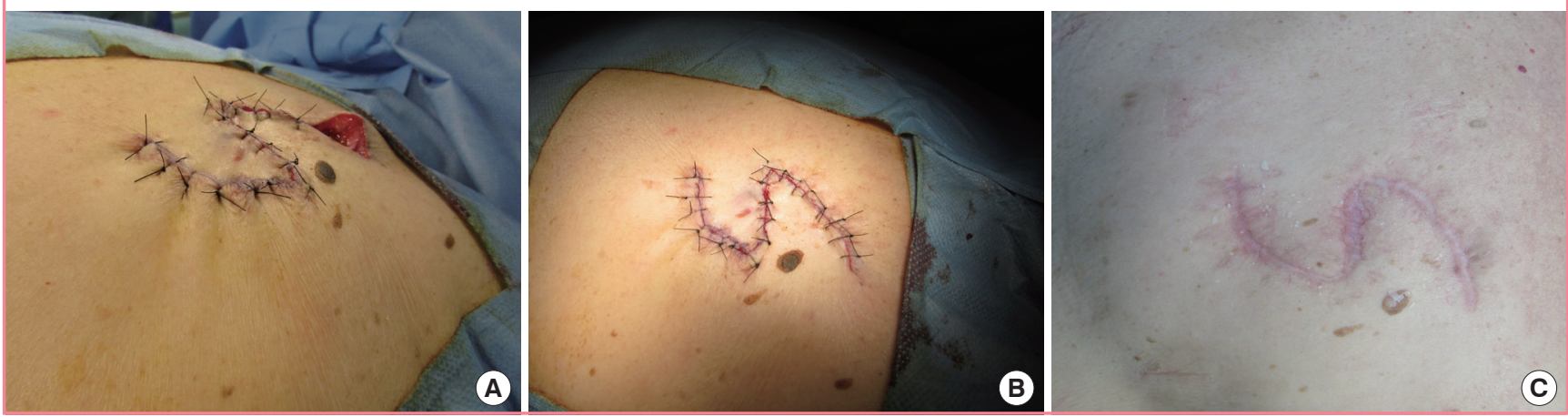

on occasion, we still have to deal with a dog ear formation.

The simplest approach to dog ears is to do nothing [5]. In some cases, in fact, the protrusion of tissue can be due to oedema and the use of local anaesthetics. However, their prominence can settle down spontaneously with time. The main risk to this method is that the dog ear might not flatten and the patient might undergo a second surgical procedure for dog ear correction. Meanwhile, patients are often disappointed at the perceived poor aesthetics in the surgical wound. A statistical analysis on dog ear regression showed that if the dog ear height is $\leq 8$ $\mathrm{mm}$, observation rather than immediate surgical removal is recommended [6].

Sometimes, dog ears may be successfully treated with de-fatting of the underlying tissue by liposuction or direct excision [7].

Since dog ears arise from length inequalities, their correction may be achieved by trying to equilibrate the side lengths in relation to the axis of the wound [8]. Realistically, this involves placing the suture such that the excess tissue redistributes along the entire length of the incision and without any protrusion [5].

Other approaches to dog ears include a variety of surgical procedures such as the straight excision with scar lengthening, $\mathrm{Bu}$ row's triangle, hockey stick and/or right-angle excision, M-plasty, S-plasty, V-Y advancement flap, and triangular advancement flap $[5,9]$.

All of these longstanding techniques have proven to be effective but require a full-thickness skin excision, resulting in a lengthened scar.

Furthermore, when dealing with flaps, correcting the dog ear can harm the blood supply to the flap by significantly reducing the pedicle size. Kishi et al. [10] described a technique that removes only the epidermis and the upper layer of the dermis, preserving the subdermal plexus.

A realistic indication for the proposed technique is a dog ear that does not exceed $15 \mathrm{~mm}$ in projection from the skin surface plane. Furthermore, within this indication limit, the ratio between the dog ear's base and its projection should not exceed 3:1.

In conclusion, the three-bite technique proved to be a fast, easy, and versatile method of immediate dog ear correction without any need to prolong scarring and with full preservation of the blood supply to the skin.

\section{REFERENCES}

1. Limberg AA. Design of local flaps. Mod Trends Plast Surg 1966;2:38-61.

2. Borgess AF. Dog ear repair. Plast Reconstr Surg 1982;69: 707-13.

3. Bennett RG. Fundamentals of cutaneous surgery. St. Louis: Mosby; 1988.

4. Gormley DE. Management of excess tissue: dog-ears, cones, and protrusio. In: Lask GP, Moy RL, editors. Principles and techniques of cutaneous surgery. New York: McGraw-Hill; 1996. p.187-99.

5. Weisberg NK, Nehal KS, Zide BM. Dog-ears: a review. Dermatol Surg 2000;26:363-70.

6. Lee KS, Kim NG, Jang PY, et al. Statistical analysis of surgical dog-ear regression. Dermatol Surg 2008;34:1070-6.

7. Chretien-Marquet B, Bennaceur S. Dog ear: true and false. A simple surgical management. Dermatol Surg 1997;23: 547-50.

8. Dzubow LM. The dynamics of dog-ear formation and closure. J Dermatol Surg Oncol 1985;11:722-8.

9. Grassetti L, Lazzeri D, Torresetti M, et al. Aesthetic refinement of the dog ear correction: the 90 degrees incision technique and review of the literature. Arch Plast Surg 2013;40: 268-9.

10. Kishi K, Nakajima H, Imanishi N. A new dog ear correction technique. J Plast Reconstr Aesthet Surg 2008;61:423-4. 